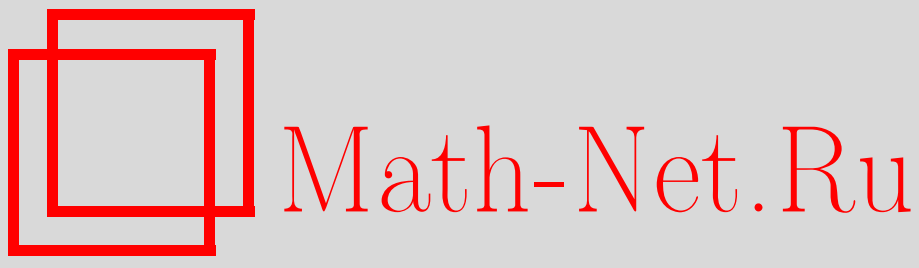

Р. Б. Гарибов, А. В. Матора, Моделирование поведения армированных элементов конструкций с учетом влияния радиационного облучения, Вестн. Сам. гос. техн. ун-та. Сер. Физ.-мат. науки, 2005, выпуск 38, 35-41

DOI: https://doi.org/10.14498/vsgtu368

Использование Общероссийского математического портала Math-Net.Ru подразумевает, что вы прочитали и согласны с пользовательским соглашением

http://www . mathnet.ru/rus/agreement

Параметры загрузки:

IP: 3.80 .253 .173

26 апреля 2023 г., 16:41:18

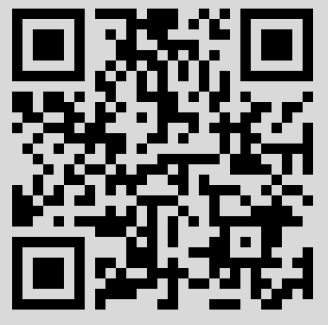




\section{МОДЕЛИРОВАНИЕ ПОВЕДЕНИЯ АРМИРОВАННЫХ ЭЛЕМЕНТОВ КОНСТРУКЦИЙ С УЧЕТОМ ВЛИЯНИЯ РАДИАЦИОННОГО ОБЛУЧЕНИЯ}

Предложена математическая модель поведения армированных элементов конструкций с учетом влияния радиационного облучения на примере деформирования прямоугольной фибробетонной пластины. Приводятся результаты расчета напряженно-деформированного состояния пластины при различных схемах воздействия и приложенной нагрузки. Показано, что под влиянием радиационной среды происходит существенное изменение механических характеристик пластины, приводящее к изменению напряженно-деформированного состояния пластины.

Экспериментальному изучению воздействия радиационных сред на металлические, бетонные, полимербетонные, железобетонные конструкции (защитные элементы реактора, ёмкости, резервуары для хранения радиоактивных отходов) посвящено определенное количество работ $[2,5,6]$.

В последнее время появились работы, посвященные математическому моделированию поведения этих конструкций в условиях воздействия радиационных сред $[1,3,4,7]$. Однако работ по моделированию работы пластинчатых конструкций из современных материалов (фибробетонов и высокопрочных железобетонов) с учетом воздействия радиационных сред весьма мало, а использованные модели взаимодействия недостаточно корректно описывают реальную работу таких элементов конструкций.

Поэтому проблема математического моделирования поведения армированных (фибробетонных, железобетонных) конструкций с учетом воздействия радиационных сред является актуальной, так как прогнозирование прочности и долговечности конструкций, длительное время находящихся в радиационной среде, имеет первостепенное значение для нормальной безаварийной эксплуатации ответственных сооружений.

В данной работе рассматривается модель деформирования прямоугольной фибробетонной пластины при воздействии радиационных сред. Эта модель может служить расчетной схемой крышки, стенки или днища прямоугольной ёмкости для хранения радиационных отходов. При построении модели использовался подход, развиваемый в [8].

Разрешающее дифференциальное уравнение изгиба фибробетонной пластины с учётом радиационного облучения имеет вид [7]:

$$
\begin{gathered}
L(W)=\frac{\partial^{2}}{\partial x^{2}}\left(D_{1} \frac{\partial^{2} W}{\partial x^{2}}\right)+\frac{\partial^{2}}{\partial x^{2}}\left(D_{2} \frac{\partial^{2} W}{\partial y^{2}}\right)+2 \frac{\partial^{2}}{\partial x \partial y}\left(D_{6} \frac{\partial^{2} W}{\partial x \partial y}\right)+\frac{\partial^{2}}{\partial y^{2}}\left(D_{2} \frac{\partial^{2} W}{\partial x^{2}}\right)+ \\
+\frac{\partial^{2}}{\partial y^{2}}\left(D_{1} \frac{\partial^{2} W}{\partial y^{2}}\right)=p(x, y)+q(x, y)
\end{gathered}
$$

где $W$ - прогиб пластины; $x, y$ - координаты в плане; $p(x, y)$ - интенсивность внешней нагрузки; $q(x, y)$ - фиктивная радиационная нагрузка, радиационная «добавка»:

$$
q=\frac{\partial^{2} \Delta M_{x}^{*}}{\partial x^{2}}+2 \frac{\partial^{2} \Delta H^{*}}{\partial x \partial y}+\frac{\partial^{2} \Delta M_{y}^{*}}{\partial y^{2}}
$$

$D_{1}, D_{2}, D_{3}, D_{4}, D_{5}, D_{6}$ - переменные жёсткости, определяющиеся из выражений

$$
\begin{gathered}
D_{1}=f_{1} J_{1}^{\phi \bar{\sigma}}+f_{2} I_{1}^{\phi \bar{\sigma}}+J_{2}^{\phi \bar{\sigma}}, D_{2}=f_{2} J_{1}^{\phi \bar{\sigma}}+f_{1} I_{1}^{\phi \bar{\sigma}}+I_{2}^{\phi \bar{\sigma}}, D_{3}=f_{3} J_{1}^{\phi \bar{\sigma}}+f_{3} I_{1}^{\phi \bar{\sigma}} ; \\
D_{4}=f_{4} J_{1}^{\phi \bar{\sigma}}-f_{5} I_{1}^{\phi \bar{\sigma}}, D_{5}=f_{4} I_{1}^{\phi \bar{\sigma}}-f_{5} J_{1}^{\phi \bar{\sigma}}, D_{6}=2 T_{2}^{\phi \bar{\sigma}}-2 \frac{\left(T_{1}^{\phi \sigma}\right)^{2}}{T_{o}^{\phi \sigma}} .
\end{gathered}
$$

В (3) используются следующие обозначения:

$$
\begin{gathered}
f_{1}=\frac{I_{0}^{\phi \bar{\sigma}} I_{1}^{\phi \bar{\sigma}}-J_{0}^{\phi \bar{\sigma}} J_{1}^{\phi \bar{\sigma}}}{\left(J_{0}^{\phi \sigma}\right)^{2}-\left(I_{0}^{\phi \sigma}\right)^{2}}, f_{2}=\frac{I_{0}^{\phi \bar{\sigma}} J_{1}^{\phi \bar{\sigma}}-J_{0}^{\phi \bar{\sigma}} I_{1}^{\phi \bar{\sigma}}}{\left(J_{0}^{\phi \sigma}\right)^{2}-\left(I_{0}^{\phi \sigma}\right)^{2}} \\
f_{3}=\frac{I_{0}^{\phi \bar{\sigma}}-J_{0}^{\phi \bar{\sigma}}}{\left(J_{0}^{\phi \bar{\sigma}}\right)^{2}-\left(I_{0}^{\phi \bar{\sigma}}\right)^{2}}, f_{4}=\frac{J_{0}^{\phi \bar{\sigma}}}{\left(J_{0}^{\phi \sigma}\right)^{2}-\left(I_{0}^{\phi \sigma}\right)^{2}}, f_{5}=\frac{I_{0}^{\phi \bar{\sigma}}}{\left(J_{0}^{\phi \sigma}\right)^{2}-\left(I_{0}^{\phi \sigma}\right)^{2}} .
\end{gathered}
$$

Жёсткости, входящие в (4), определяются формулами 


$$
\begin{gathered}
J_{k}^{\phi \sigma}=\int_{-h / 2}^{z_{0}} \alpha_{j} z^{k} \partial z+\int_{z_{0}}^{h / 2} \alpha_{i} z^{k} \partial z, \text { при } k=0,1,2 ; \quad I_{k}^{\phi \sigma}=\int_{-h / 2}^{z_{0}} \alpha_{j} v_{j} z^{k} \partial z+\int_{z_{0}}^{h / 2} \alpha_{i} v_{i} z^{k} \partial z, \text { при } k=0,1,2 ; \\
T_{k}^{\phi \sigma}=\int_{-h / 2}^{z_{0}} \beta_{j} z^{k} \partial z+\int_{z_{0}}^{h / 2} \beta_{i} z^{k} \partial z, \text { при } k=0,1,2 .
\end{gathered}
$$

Здесь

$$
\begin{gathered}
z_{0}=-\frac{\varepsilon_{x}+\varepsilon_{y}}{\chi_{x}+\chi_{y}}+\frac{2\left(1-v_{c p}^{2}\right) \cdot \varepsilon_{\Phi}^{\phi \sigma}}{\chi_{x}+\chi_{y}} ; \\
\alpha_{j}=\frac{\Psi_{j}}{1-v_{j}^{2}} ; \alpha_{i}=\frac{\Psi_{i}}{1-v_{i}^{2}} ; \beta_{j}=\frac{\Psi_{j}}{2\left(1+v_{j}\right)} ; \beta_{i}=\frac{\Psi_{i}}{2\left(1+v_{i}\right)} v_{c p}=\frac{v_{j}+v_{i}}{2} .
\end{gathered}
$$

В этих выражениях $z_{0}-$ уравнение нейтральной поверхности, отделяющей растянутую зону пластинки от сжатой; $i, j-$ индексы, характеризующие сжатую и растянутую зону пластинки. При растянутой нижней зоне изгибаемой пластинки $j=1, i=2$; при растянутой верхней зоне и сжатой нижней $j=2, i=1 ; h$ - толщина пластины.

В данной работе используется следующее выражение для секущего модуля:

$$
\psi_{j}^{\phi \bar{\sigma}}=\left\{\begin{array}{l}
\left(A_{j}-B_{j} e_{u}^{2}\right), \Phi<\Phi_{1 \text { nop }}, \\
\left(A_{j}-B_{j} e_{u}^{2}\right) \cdot f(\Phi), \Phi \geq \Phi_{1 \text { nop }},
\end{array}\right.
$$

где $f(\Phi)=1-\alpha_{1} \lg \left(\beta_{1} \cdot \Phi\right)$,

$$
\begin{gathered}
e_{u}=\frac{\sqrt{2}}{2\left(1+v_{j}^{\phi \sigma}\left(e_{u}, \Phi\right)\right)} \sqrt{\left(e_{x}-e_{y}\right)^{2}+e_{x}^{2}+e_{y}^{2}+\frac{2}{3}\left(e_{x y}^{2}\right)}, \\
e_{x}=\varepsilon_{x}+\chi_{x} z ; e_{y}=\varepsilon_{y}+\chi_{y} z ; e_{x y}=\varepsilon_{x y}+2 \chi_{x y} z .
\end{gathered}
$$

Зависимость, описывающая изменение радиационного распухания, задается соотношением

$$
\varepsilon_{\Phi}^{\phi \sigma}=\left\{\begin{array}{l}
0, \Phi<\Phi_{2 \text { nop }}, \\
\delta \cdot \varepsilon_{\max }\left[\exp \left(v \cdot\left(\Phi-\Phi_{2 \text { nop }}\right)\right)-1\right] /\left[\varepsilon_{\max }+\delta \exp \left(v \cdot\left(\Phi-\Phi_{2 \text { nop }}\right)\right)\right], \Phi \geq \Phi_{2 \text { nop }},
\end{array}\right.
$$

где $\varepsilon_{\max }$ - максимальное значение радиационных деформаций для бетона данного состава; $\varepsilon_{\phi}$ - радиационные деформации бетона или радиационное распухание (объёмное изменение); $\delta, v$ - эмпирические коэффициенты, зависящие от радиационной деформативности заполнителя и энергетического спектра потока нейтронов; $\Phi$ - доза облучения или (флюенс); $\Phi_{1 \text { nор }}, \Phi_{2 \text { пор }}-$ пороговые дозы облучения, по достижению которых начинается изменение механических характеристик и появляется распухание; $\varepsilon_{x}, \varepsilon_{y}, \varepsilon_{x y}$ - деформация точек срединной поверхности; $\chi_{x}, \chi_{y}, \chi_{x y}$ - кривизны в этих точках; $z$ - координаты рассматриваемых точек, отсчитываемые от срединной поверхности; $v_{j}$ - коэффициент поперечной деформации; $e_{u}$ интенсивность деформаций точки.

Моделирование поведения пластины в условиях радиационного облучения можно проводить при разных программах и схемах нагружения.

Схема нагружения определяет закон распределения внешней нагрузки по верхней или нижней поверхностям пластины, а также величину нормальных усилий на ее торцевых гранях. Программа нагружения определяет характер (кинетику) изменения нагрузки, действующей на пластину во времени.

Рассмотрим нижеследующую программу работы пластины.

1. Пластина нагружается распределенной статической нагрузкой по верхней поверхности до определенного уровня. Воздействие внешних усилий на торцевые грани пластины отсутствует. Влияние радиационного поля отсутствует. В этом случае в пластине устанавливается определенное напряженно-деформированное состояние.

2. По всей верхней поверхности пластины действует радиационное облучение, в результате с течением времени доза облучения в каждой точке пластины изменяется, приводя к появлению наведенной неоднородности механических свойств и радиационному распуханию материала пластины. Под влиянием этих факторов происходит перераспределение напряжений и деформаций по объему пластины.

3. При достижении напряжениями или деформациями опасного уровня (при наступлении 
предельного состояния какого-либо вида) считается, что пластина выходит из строя.

Алгоритм расчета пластины включает несколько блоков: блок задания исходных данных; блок силового расчета пластины на действие нагрузки без учета радиационного облучения; блок определения напряженно-деформированного состояния и проверки прочности пластины; блок расчета закона распределения дозы и радиационного распухания по объему пластины; блок расчета пластины с учетом облучения; блок определения напряженно-деформированного состояния при совместном действии нагрузки и облучения; блок проверки выполнения условий прочности и долговечности.

В алгоритме заложена процедура итерационного уточнения, позволяющая получать достаточно точные результаты при ограниченном числе циклов. При определении закона распределения дозы по толщине (объему) пластины используется зависимость

$$
\Phi(x, y, z)=\Phi_{0} \exp \left[-\frac{(h / 2)-z}{L}\right],
$$

которая отнесена к системе координат, связанной со срединной поверхностью пластины. В этой формуле значение дозы $\Phi_{0}$ в поверхностных слоях пластины определяется в соответствии с зависимостью

$$
\Phi_{0}=I_{(t)} \cdot t,
$$

где $I_{(t)}$ - интенсивность облучения, $t-$ время.

С использованием данного алгоритма был разработан программный комплекс, реализованный на базе пакета математических и инженерных задач Matlab и предназначенный для выполнения статических расчетов плоских фибробетонных элементов конструкций (расчетная схема которых сводится к прямоугольной пластине) на действие нагрузки и радиационного облучения. Аппроксимация уравнений, описывающих деформирование рассчитываемых конструкций, производится методом сеток.

Программный комплекс позволяет исследовать напряженно-деформированное состояние фибробетонной пластины при совместном действии нагрузки и радиационного облучения при различных схемах и сроках воздействия радиации и внешней приложенной нагрузки. Схемы воздействия радиационного поля и приложенной внешней нагрузки могут быть следующими: радиация и нагрузка действуют совместно на верхнюю сторону пластины; радиация действует на нижнюю сторону пластинки, а нагрузка на верхнюю; пластина лежит на упругом основании и находится под совместным воздействием нагрузки и радиационного облучения; пластина подвергается воздействию неравномерно распределённой и радиационному облучению.

Ниже в табл. 1-4 приведены результаты расчёта фибробетонной пластины при разных схемах воздействия радиации и приложенной нагрузки.

\section{Результаты расчётов пластины при однонаправленном

\begin{tabular}{|c|c|c|c|c|c|c|}
\hline \multicolumn{3}{|c|}{$\begin{array}{c}\text { Схема воздействия внешних } \\
\text { факторов }\end{array}$} & \multicolumn{4}{|c|}{$\downarrow^{\text {Rad }}$} \\
\hline 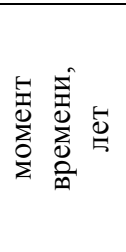 & 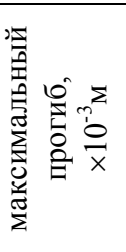 & 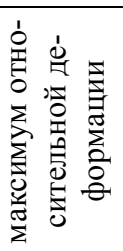 & 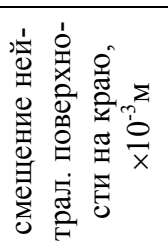 & 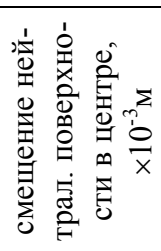 & 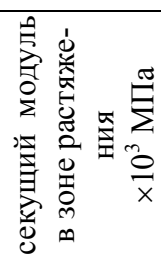 & 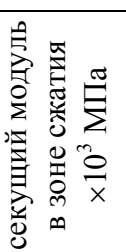 \\
\hline 1 & 2 & 3 & 4 & 5 & 6 & 7 \\
\hline 0 & 0.579 & 0.848 & 2.827 & -1.739 & 23.52 & 36.18 \\
\hline 1 & 2 & 3 & 4 & 5 & 6 & 7 \\
\hline 1 & 0.578 & 0.848 & 2.645 & -1.909 & 23.55 & 36.18 \\
\hline 3 & 0.575 & 0.843 & 2.489 & -2.087 & 23.63 & 36.19 \\
\hline 6 & 0.618 & 0.912 & 4.080 & -0.585 & 18.26 & 36.17 \\
\hline 10 & 0.710 & 1.040 & 5.318 & -0.330 & 13.65 & 31.94 \\
\hline 15 & 0.811 & 1.196 & 6.820 & -0.080 & 9.973 & 28.07 \\
\hline 21 & 0.923 & 1.391 & 8.554 & 0.202 & 7.291 & 24.86 \\
\hline 30 & 1.089 & 1.686 & 10.99 & 0.587 & 4.890 & 21.45 \\
\hline
\end{tabular} действии нагрузки и радиационного облучения}


Результаты расчётов пластины при разнонаправленном действии нагрузки и радиационного облучения

\begin{tabular}{|c|c|c|c|c|c|c|}
\hline \multicolumn{3}{|c|}{$\begin{array}{c}\text { Схема воздействия внешних } \\
\text { факторов }\end{array}$} & \multicolumn{4}{|c|}{$\underbrace{1+1+1+1+1+1}$} \\
\hline 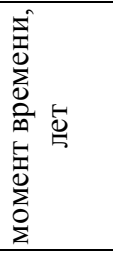 & 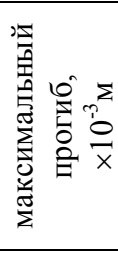 & 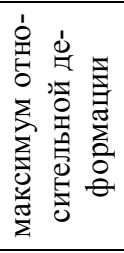 & 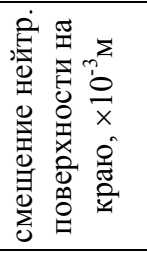 & 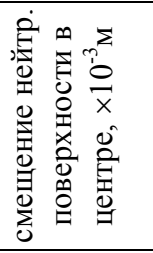 & 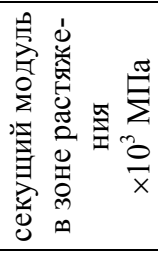 & 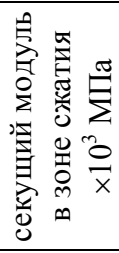 \\
\hline 0 & 0.578 & 0.848 & 2.827 & -1.739 & 23.32 & 36.18 \\
\hline 1 & 0.578 & 0.849 & 2.985 & -1.523 & 23.32 & 36.18 \\
\hline 3 & 0.575 & 0.847 & 3.135 & -1.355 & 23.36 & 36.18 \\
\hline 6 & 0.623 & 0.886 & 1.867 & -2.573 & 22.64 & 29.71 \\
\hline 10 & 0.716 & 0.995 & 2.053 & -3.321 & 18.17 & 24.85 \\
\hline 15 & 0.818 & 1.121 & 2.396 & -4.049 & 14.21 & 20.99 \\
\hline 21 & 0.934 & 1.284 & 2.992 & -4.732 & 11.01 & 17.78 \\
\hline 30 & 1.108 & 1.534 & 3.989 & -5.611 & 7.984 & 14.37 \\
\hline
\end{tabular}

Т а б л и ц а 3

Результаты расчётов пластины лежащей на упругом основании, под действием нагрузки и радиационного облучения

\begin{tabular}{|c|c|c|c|c|c|c|}
\hline \multicolumn{3}{|c|}{$\begin{array}{c}\text { Схема воздействия внешних } \\
\text { факторов }\end{array}$} & \multicolumn{4}{|c|}{ 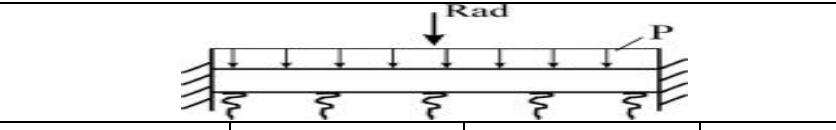 } \\
\hline 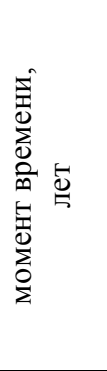 & 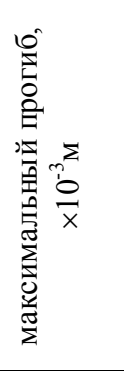 & 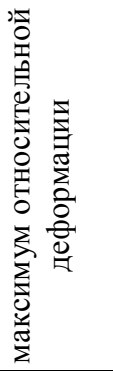 & 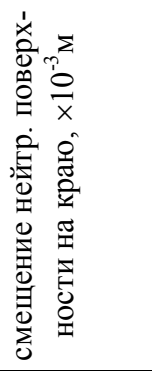 & 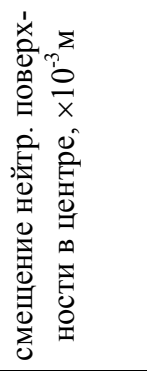 & 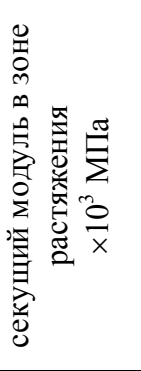 & 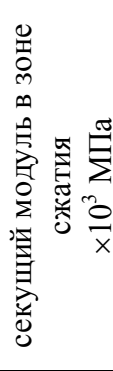 \\
\hline 0 & 0.339 & 0.553 & 2.088 & -1.641 & 27.57 & 36.33 \\
\hline 1 & 0.339 & 0.552 & 1.876 & -1.936 & 27.60 & 36.33 \\
\hline 3 & 0.336 & 0.550 & 1.710 & -2.244 & 27.61 & 36.33 \\
\hline 6 & 0.350 & 0.577 & 3.003 & -0.544 & 22.43 & 36.32 \\
\hline 10 & 0.377 & 0.621 & 3.799 & -0.225 & 18.43 & 32.11 \\
\hline 15 & 0.402 & 0.663 & 4.590 & 0.116 & 15.28 & 28.27 \\
\hline 21 & 0.427 & 0.713 & 5.336 & 0.460 & 12.63 & 25.07 \\
\hline 30 & 0.458 & 0.779 & 6.319 & 0.958 & 9.858 & 21.69 \\
\hline
\end{tabular}

Рассмотрим более детально случай, когда радиация и нагрузка действуют совместно на верхнюю сторону пластины при сроке воздействия от 0 до 30 лет. При расчёте использовалась зависимость вида (8), которая имеет следующие параметры: начальные коэффициенты для растяжения $A_{10}=30.71 \cdot 10^{3} \mathrm{MПа} ; B_{10}=11.79 \cdot 10^{10} \mathrm{MПа;} \mathrm{начальные} \mathrm{коэффициенты} \mathrm{для} \mathrm{сжатия}$ $A_{20}=36.44 \cdot 10^{3} \mathrm{MПа;} B_{20}=46.20 \cdot 10^{8}$ Мпа; $E_{0}=33 \cdot 10^{3} \mathrm{MПа} \mathrm{-} \mathrm{модуль} \mathrm{упругости} \mathrm{определённый} \mathrm{по}$ диаграмме деформирования фибробетона (в не радиационных условиях).

Геометрические размеры пластины: ширина 2 м, длина 3 м, толщина пластины 0.12 м. Параметры радиационного воздействия: $I=3.3 \cdot 10^{19}$ нейтрон/( $\mathrm{cm}^{2} \cdot$ год $)$, интенсивность нейтронного облучения; $\alpha_{1}=0.6, \beta_{1}=1 \cdot 10^{-20} \mathrm{~cm}^{2} /$ нейтрон, $L=0.16 \mathrm{м}$ - длина диффузии, зависящая от энергии нейтронов; $\Phi_{1 \text { пор }}=1 \cdot 10^{20}$ нейтрон $/ \mathrm{cm}^{2}$, пороговая доза облучения для $\psi_{j}, E_{j} ; \Phi_{2 \text { пор }}=1 \cdot 10^{19}$ нейтрон/см², пороговая доза облучения для радиационной деформации распухания $\varepsilon_{\Phi}$; $\varepsilon_{\text {max }}=1 \cdot 10^{-4}$ — максимальные деформации в формуле (11) для нахождения $\varepsilon_{\Phi} ; \delta=0,5$ - коэффициент в формуле $(11) ; v=1 \cdot 10^{-20} \mathrm{~cm}^{2} /$ нейтрон, коэффициент в формуле (11) $p-$ нагрузка 0.08 МПа. 


\section{Результаты расчётов пластины под действием неравномерной} нагрузки и радиационной среды

\begin{tabular}{|c|c|c|c|c|c|c|}
\hline \multicolumn{3}{|c|}{$\begin{array}{c}\text { Схема воздействия внешних } \\
\text { факторов }\end{array}$} & \multicolumn{4}{|c|}{ Rad } \\
\hline 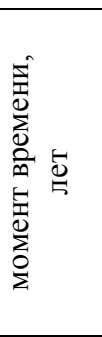 & 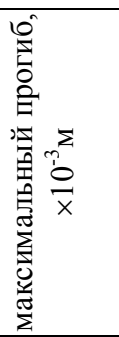 & 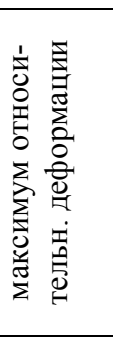 & 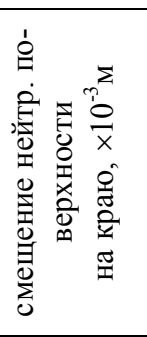 & 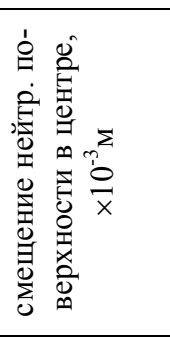 & 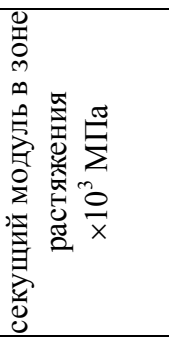 & 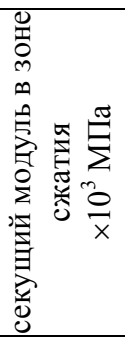 \\
\hline 0 & 0.583 & 1.038 & 3.595 & -1.702 & 19.67 & 36.06 \\
\hline 1 & 0.583 & 1.041 & 3.428 & -1.888 & 19.65 & 36.07 \\
\hline 3 & 0.580 & 1.035 & 3.261 & -2.082 & 19.77 & 36.07 \\
\hline 6 & 0.624 & 1.132 & 5.124 & -0.550 & 14.85 & 36.05 \\
\hline 10 & 0.717 & 1.315 & 6.835 & -0.281 & 10.72 & 31.81 \\
\hline 15 & 0.818 & 1.528 & 8.734 & -0.015 & 7.814 & 27.94 \\
\hline 21 & 0.933 & 1.768 & 10.70 & 0.287 & 5.737 & 24.73 \\
\hline 30 & 1.102 & 2.140 & 13.34 & 0.705 & 3.851 & 21.32 \\
\hline
\end{tabular}

Представляет определённый интерес проследить, как меняются во времени некоторых механических характеристик пластинки.

На рис. 1 показаны эпюры дозы облучения $\Phi$ по координате $z$ для разных моментов времени $t=0,5,10,15,20,25,30$ лет, для случая, когда радиация действует на верхнюю поверхность пластинки ( $z=-0.06 \mathrm{M})$.

На рис. 2 приведена кинетика величины $\varepsilon_{\Phi}$ (радиационное распухание) во времени для центральной точки на верхней поверхности пластинки (сплошная линия) и нижней поверхности (пунктир). Аналогичные кинетические зависимости для параметра $f(\Phi)$, определяющего деградацию секущего модуля, показаны на рис. 3. По этим графикам можно примерно определить инкубационный период каждого процесса.

На рис. 4 показаны изменения максимального прогиба. Как видим, в период до 3 лет прогиб убывает, что мы связываем с действием радиационного распухания, а затем монотонно возрастает из-за доминирующего влияния деградации секущего модуля. На рис. 5. приводится кинетика максимальной по объему пластинки относительной интенсивности деформаций. Превышение предельного уровня, отмеченного пунктиром, происходит в момент времени $t=9$ лет.

Обе рассмотренные характеристики за весь срок воздействия радиации возрастают примерно вдвое.

На рис. 6 и 7 показано изменение во времени положения нейтральной поверхности $z_{0}$ для разных точек пластинки в разные моменты времени. В подтверждение ранее приводимых результатов, нейтральная поверхность смещается вверх до $t=3$ года, пока не начнется процесс деградации секущего модуля, после этого срока происходит монотонное движение вниз.

Кинетика деградации секущего модуля по толщине для центра пластинки приведена на рис. 8, а для середины длинного края - на рис. 9. На каждом графике имеется по 7 эпюр для равноотстоящих моментов времени $t=0,2$, 4, 6, 8, 10, 12 лет. Первые две эпюры практически сливаются друг с другом.

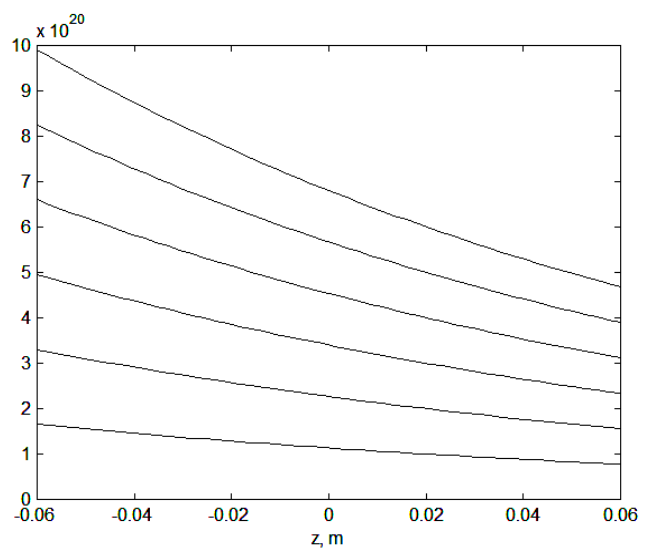

Р и с. 1. Эпюры дозы облучения Ф от координаты $z$ для разных моментов времени $(\mathrm{t}=0,5,10,15,20,25,30$ лет) 


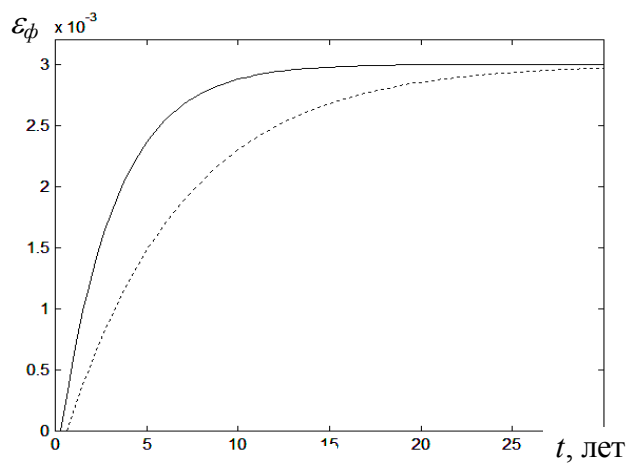

Р и с. 2. Кинетика величины $\varepsilon_{\Phi}$ от времени для центральной точки на верхней стороне пластинки (сплошная линия) и нижней стороне (пунктир)

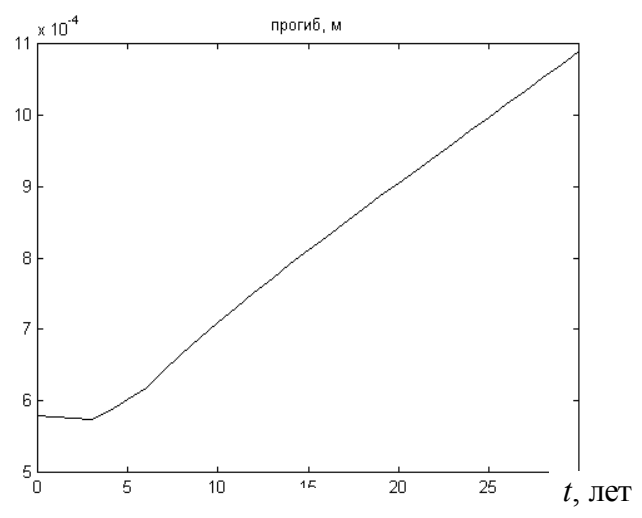

Р и с. 4. Зависимость максимального прогиба пластинки от времени

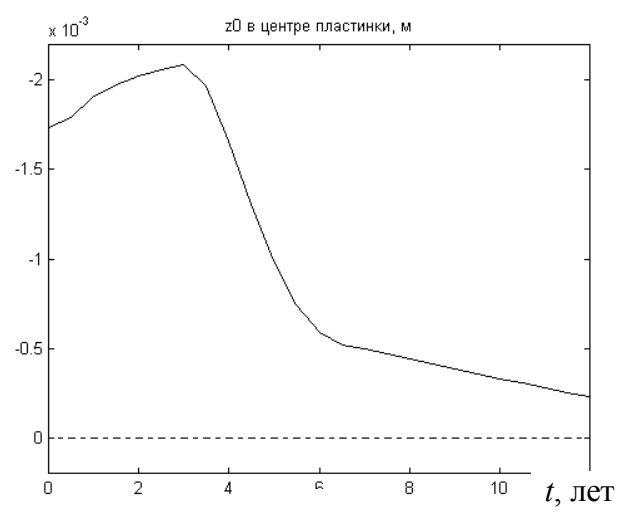

Р и с. 6. Изменение во времени положения нейтральной поверхности $z_{0}$ для центра пластинки $(t=0,2,4,6,8,10,12)$

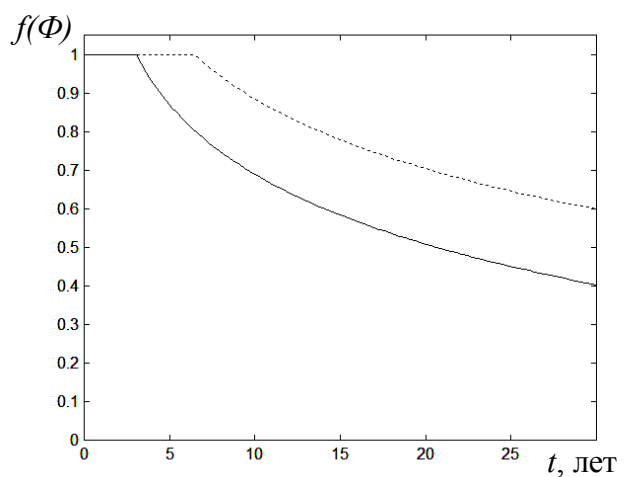

Р и с. 3. Кинетическая зависимость для параметра $f(\Phi)$, определяющего деградацию секущего модуля $\psi$ для верхней стороны пластинки (сплошная линия) и нижней стороны (пунктир)

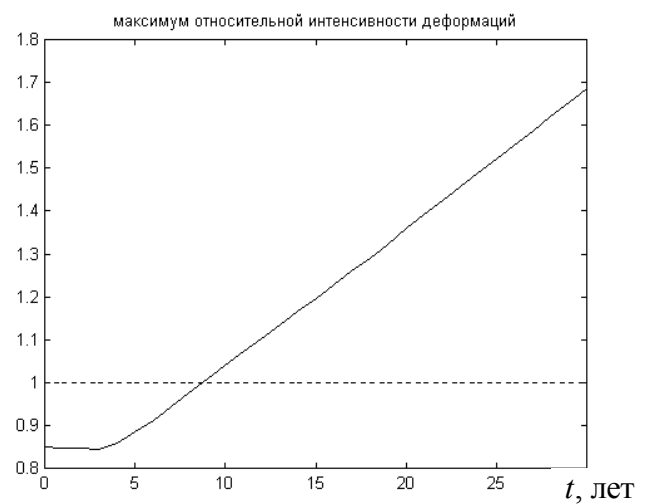

Р и с. 5. Кинетика максимальной относительной интенсивности деформаций по объёму пластинки

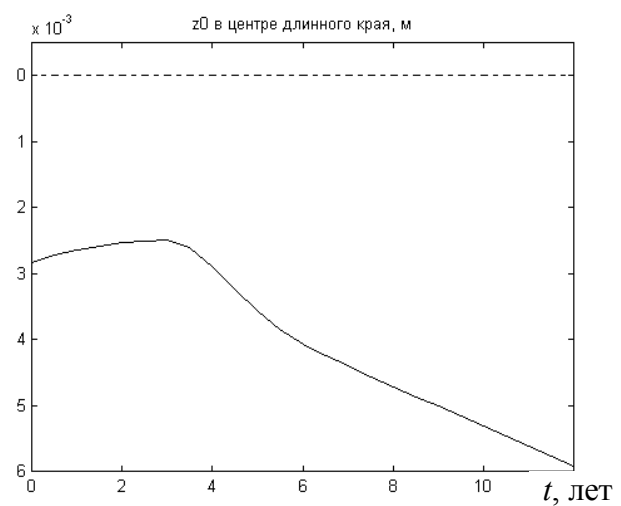

Р и с. 7. Изменение во времени положения нейтральной поверхности $z_{0}$ для середины длинного края пластинки $(t=0,2,4,6,8,10,12)$ 


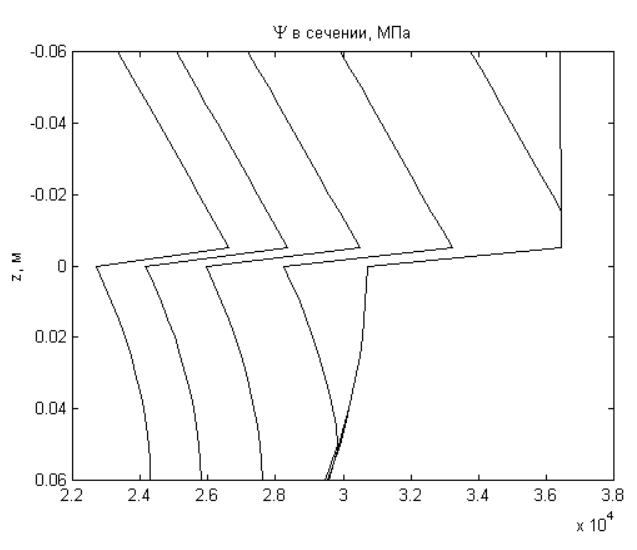

Р и с. 8. Деградация секущего модуля во времени по толщине для центра пластинки $(t=0,2,4,6,8,10,12)$

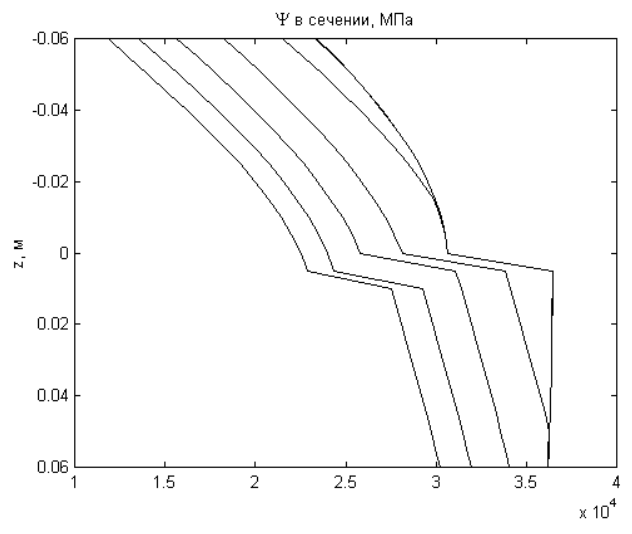

Р и с. 9. Деградация секущего модуля во времени по толщине, для середины длинного края пластинки $(t=0,2,4,6,8,10,12)$

Расчёты показывают, что с течением времени, под влиянием радиационной среды происходит значительное изменение механических характеристик материала пластины, приводящее к изменению напряженно-деформированного состояния пластины.

\section{БИБЛИОГРАФИЧЕСКИЙ СПИСОК}

1. Андреев В. И. Некоторые задачи и методы механики неоднородных тел М.: Изд. АСВ, 2002. 288 с.

2. Гусев Н. Г. Защита от ионизирующих излучений / Н. Г. Гусев, В. А. Климанов, В. П. Машкович. Т. 1. М.: Энергоатомиздат, 1989. $509 \mathrm{c.}$

3. Дубровская E. В. Некоторые плоские и осесимметричные задачи равновесия упругого тела находящегося в условиях радиационного облучения. Саратов. Автореф. дисс. ... канд. техн. наук. 1989. 17 с.

4. Дубровский A. B. Исследование напряжённо-деформированного состояния строительных конструкций, находящихся под воздействием ионизирующих излучений. Саратов. Автореф. дисс. ... канд. техн. наук. 1986. $21 \mathrm{c}$.

5. Дубровский A. В. Радиационная стойкость строительных материалов. М.: Стройиздат, 1977. 278c.

6. Дубровский В. Б. Строительные материалы и конструкции защиты от ионизирующего излучения / В. Б. Дубровский, 3. Аблевич. М.: Стройиздат, 1983. 240 с.

7. Овчинников И. Г. Напряженно-деформированное состояние армированных элементов конструкций при воздействии радиационных полей / И. Г. Овчинников, А. В. Матора, Г. А. Наумова. Саратов: СГТУ. 2004. 204 с.

8. Гарибов Р. Б. Сопротивление железобетонных элементов конструкций воздействию агрессивных сред. Саратов: СГУ, 2003. $228 \mathrm{c}$.

Поступила 12.04.2005 2. 